\title{
Technè
}

La science au service de l'histoire de l'art et de la préservation des biens culturels

41 | 2015

Arts textiles antiques et modernes. Approche scientifique et restauration

\section{La Dame à la licorne, sa conservation et l'évaluation colorimétrique du nettoyage}

"The Lady and the Unicorn" tapestries, their conservation and the colorimetric assessment of the cleaning technique

Élisabeth Taburet-Delahaye, Raphaëlle Déjean, Dominique de Reyer et Witold Nowik

\section{OpenEdition}

Journals

Édition électronique

URL : http://journals.openedition.org/techne/4758

DOI : $10.4000 /$ techne.4758

ISSN : 2534-5168

Éditeur

C2RMF

Édition imprimée

Date de publication : 25 novembre 2015

Pagination : 86-93

ISBN : 978-2-7118-6248-1

ISSN : $1254-7867$

\section{Référence électronique}

Élisabeth Taburet-Delahaye, Raphaëlle Déjean, Dominique de Reyer et Witold Nowik, «La Dame à la licorne, sa conservation et l'évaluation colorimétrique du nettoyage », Technè [En ligne], 41 | 2015, mis en ligne le 08 août 2020, consulté le 11 mars 2021. URL : http://journals.openedition.org/techne/4758 ; DOI : https://doi.org/10.4000/techne.4758

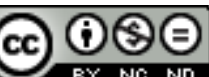

La revue Technè. La science au service de l'histoire de l'art et de la préservation des biens culturels est mise à disposition selon les termes de la Licence Creative Commons Attribution - Pas d'Utilisation Commerciale - Pas de Modification 4.0 International. 


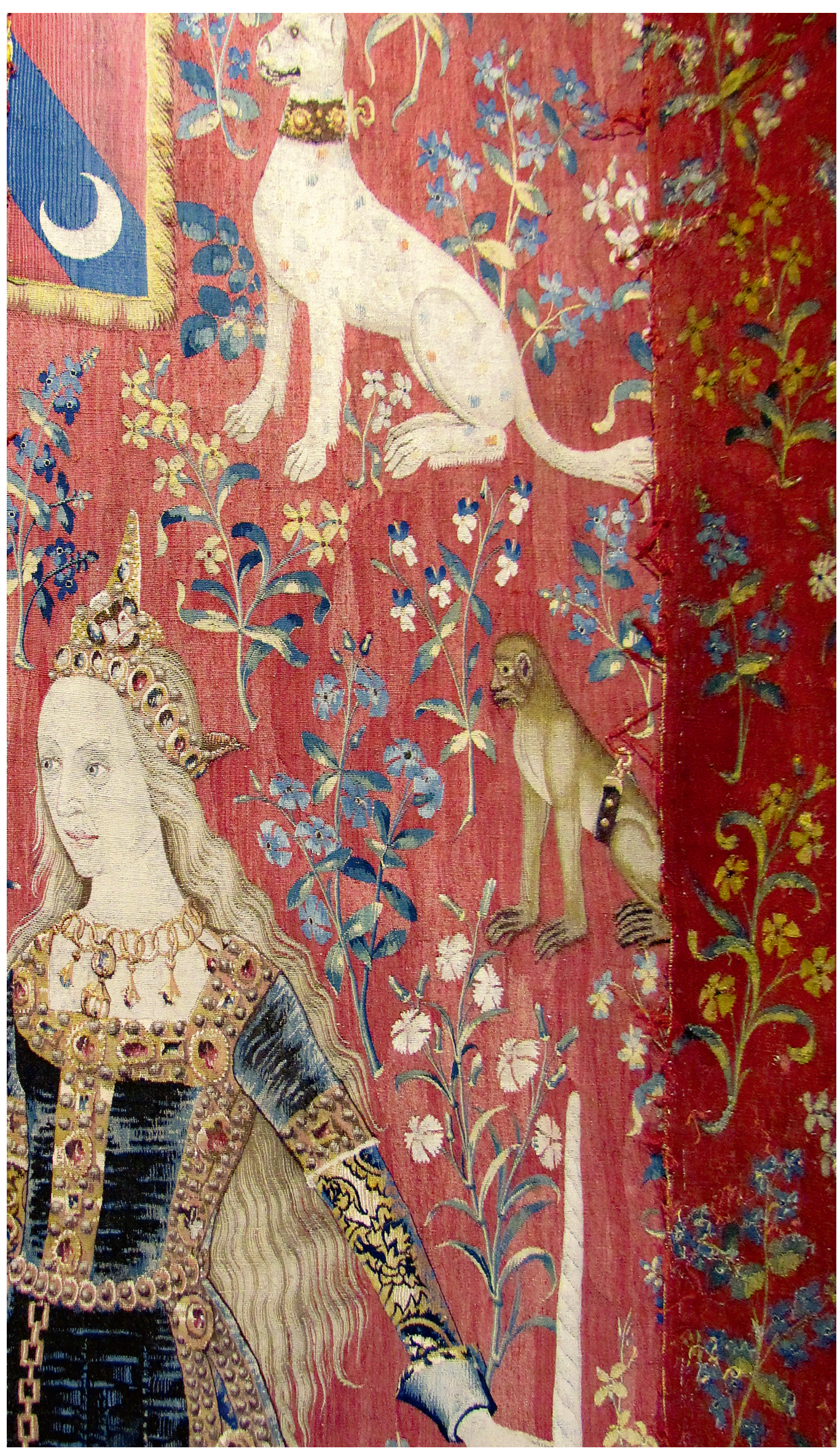

Fig. 1. Exemple de la

différence de décoloration des coloris originaux entre la face (à gauche) et le revers (à droite) de la tapisserie "Le Toucher " @ C Raphaëlle Déjean. 
Élisabeth Taburet-Delahaye

Raphaëlle Déjean

Dominique de Reyer

Witold Nowik

\section{La Dame à la licorne, sa conservation et l'évaluation colorimétrique du nettoyage}

"The Lady and the Unicorn" tapestries, their conservation and the colorimetric assessment of the cleaning technique
Résumé. Les six tapisseries de la tenture de La Dame à la licorne forment un ensemble exceptionnel à bien des égards : une iconographie abondamment commentée, une histoire mouvementée, une qualité d'exécution remarquable. Malgré la grande vigilance déployée à leur égard, leur état nécessitait des interventions de conservation qui devaient également orienter les choix muséographiques de la salle rénovée. Des analyses colorimétriques ont permis d'apprécier l'efficacité du lavage préconisé en raison de leur état sanitaire, mais également de définir un référentiel pour le suivi futur de leur état de surface.

Mots-clés. Tapisserie, conservation, restauration, lavage, colorimétrie.
Abstract. The six tapestries of The Lady and the Unicorn series form an outstanding ensemble in many respects: the largely studied iconography, the eventful history and the remarkable quality of their execution. Although well taken care of, their condition required some conservation treatment, which would also determine the display conditions in the renovated room. Colorimetric analyses enabled us not only to assess the efficiency of the washing technique, recommended for the tapestries for conservation reasons, but also to collect the set of reference values for the monitoring of future changes on the tapestries surface.

Keywords. Tapestry, conservation, restoration, cleaning, washing, colorimetry.

\section{Introduction}

Mentionnée au château de Boussac depuis le début du XIX ${ }^{\mathrm{e}}$ siècle, signalée en 1841 par Prosper Mérimée, alors jeune inspecteur des Monuments historiques, citée par George Sand à plusieurs reprises, La Dame à la licorne fut acquise par l'État au profit du musée de Cluny en 1882. Pour Edmond Du Sommerard, directeur du musée depuis son ouverture en 1844, il s'agissait de l'aboutissement de longues négociations avec la ville de Boussac, propriétaire depuis 1837 du château où elle logea la sous-préfecture dès 1838 .

Avant même l'acquisition de la tenture par le musée de Cluny, les armoiries avaient été reconnues comme celles d'une famille de robe d'origine lyonnaise, les Le Viste. L'iconographie des six pièces a suscité - et suscite encore - hypothèses et controverses, mais l'identification de cinq d'entre elles comme figurant les allégories des cinq sens a été le plus souvent retenue $^{1}$. Le milieu d'origine des modèles a été précisé, notamment grâce à Nicole Reynaud qui a proposé l'attribution de la tapisserie au Maître des Très Petites Heures d'Anne de Bretagne, très probablement Jean d'Ypres, peintre actif à Paris entre 1490 et $1508^{2}$. Selon plusieurs éléments pris en compte par les différents auteurs, la commande effectuée peu après 1500 reviendrait à Antoine II $^{3}$.
Les dégradations subies par les tapisseries au XIX ${ }^{\mathrm{e}}$ siècle furent signalées par Prosper Mérimée et George Sand, cette dernière mentionnant une restauration à Aubusson dont nous ne savons rien - pas même si elle eut réellement lieu. Dès son acquisition par l'État, la tenture fut envoyée aux Gobelins pour restauration. En 1883-1884, les parties basses fortement endommagées furent remplacées par des toiles peintes, auxquelles Alfred Darcel, nommé à la tête du musée en 1885 , décida, en 1889, de substituer un retissage.

Pendant la Seconde Guerre mondiale, le musée étant fermé, une nouvelle intervention fut menée aux Gobelins sous la surveillance d'une commission réunie par le conservateur François de Montrémy. Celle-ci autorisa quelques repiquages et retissages, accompagnés de la pose d'une doublure et de la mise en place d'un nouveau galon.

Dans la seconde moitié du $\mathrm{Xx}^{\mathrm{e}}$ siècle, plusieurs interventions plus limitées relevèrent avant tout de la conservation ${ }^{4}$.

En dépit de cette succession de soins, l'examen des tapisseries mené en 2012 confirma les inquiétudes suscitées par l'observation à l'œil nu. En effet, l'empoussièrement, les tensions et les déformations étaient de plus en plus manifestes. Par ailleurs, le système d'éclairage complexe mis en place à l'occasion du réaménagement de la rotonde en 1992-1993 avait perdu de son efficacité et ne permettait plus un

Élisabeth Taburet-Delahaye, conservateur général, directrice du musée de Cluny-Musée national du Moyen Âge, Paris (elisabeth.taburetdelahaye@culture.gouv.fr). Raphaëlle Déjean, restauratrice de textiles, Atelier Curial, Paris (raphaelledejean@yahoo.fr). Dominique de Reyer, ingénieur de recherche, LRMH, Champs-sur-Marne (dominique.de-reyer@culture.gouv.fr). Witold Nowik, chimiste, ingénieur de recherche, département Recherche, C2RMF, Paris (witold.nowik@culture.gouv.fr). 
éclairement régulier. Il fut donc décidé de mener une opération de conservation-restauration, prélude à la rénovation de la muséographie dont l'intervention devait également dresser le cahier des charges.

\section{État de conservation et protocole de conservation}

Si son histoire et son iconographie font de cette tenture une œuvre exceptionnelle, la qualité de son exécution est également remarquable. Dans les parties originelles, les six tapisseries révèlent un tissage d'une grande finesse ${ }^{5}$ et d'une grande maîtrise technique ${ }^{6}$. Le raffinement passe également par l'emploi d'une large gamme colorée ${ }^{7}$ pour les fils de trame en laine ou en soie. En effet, tandis que les chaînes sont en laine écrue, les trames présentent des couleurs aux nuances subtiles, obtenues avec des colorants naturels dont chaque teinte est déclinée en trois tons : un clair, un moyen et un foncé. Ces colorants sont ceux couramment employés à l'époque : la garance pour les rouges, la gaude pour les jaunes, le pastel pour les bleus, plusieurs tanins pour les bruns, mais on trouve aussi, fait plus rare, de l'orseille (substance colorante issue de lichens) pour les bruns violacés ${ }^{8}$.

Les parties basses retissées ont, quant à elles, de toutes autres caractéristiques, qui expliquent largement leur différence d'aspect. Leur tissage est beaucoup plus grossier, les chaînes sont en coton blanc et les trames exclusivement en laine sont, pour la plupart, chinées. Les colorants employés sont des colorants synthétiques ${ }^{9}$. Ces colorants ont mal résisté à la lumière et les parties basses sont aujourd'hui nettement plus décolorées que les parties originelles qui ont pourtant pâli sur la face au cours de leur exposition, ainsi que le prouve l'intensité des couleurs au revers (fig. 1). Comme souvent, les composantes jaunes des teintes vertes ont disparu, dégageant une dominante bleue des végétaux. L'orseille, colorant fragile, a pour sa part complètement viré au bleu clair.

L'empoussièrement et l'encrassement constatés sur la tenture sont importants et ils ont achevé de la ternir. Bien que variables d'une tapisserie à l'autre ${ }^{10}$, ils ont altéré à la fois leur aspect et leurs fibres, devenues sèches, sales et cassantes.

D'un point de vue structurel, d'importantes tensions et déformations ont été également révélées par un éclairage en lumière rasante. Elles résultent de la combinaison de plusieurs facteurs : une présentation sur des murs incurvés, de fortes tensions le long du velcro de suspension, des parties basses contraignantes, mais surtout des doublures inadaptées. La toile utilisée pour ces doublages est lourde et raide, et elle a été fixée selon la technique traditionnelle du carroyage ${ }^{11}$ qui bloque le mouvement des tensions verticales de la tapisserie.

Enfin, les tissages présentaient de petites zones de faiblesse : des relais ouverts, des parties où les trames étaient usées ou manquantes, quelques ruptures de chaînes et des tensions locales dues aux anciennes restaurations.
Le constat de ces altérations a conduit à l'élaboration d'un protocole de conservation pour l'ensemble de la tenture, avec, au cour de nos réflexions, la volonté d'une réelle pérennité de nos interventions et les questions soulevées par le nettoyage d'une œuvre aussi prestigieuse.

Chaque pièce a donc été décrochée, puis son ancienne doublure retirée. Le dépoussiérage a ensuite été effectué par micro-aspiration de la face et du revers.

Certaines anciennes restaurations ont été allégées lors de la préparation des tapisseries pour le lavage. Pour éviter toute contention et permettre une bonne remise à plat, les fils courant d'un repiquage à un autre ont été coupés, certains nœuds ou renchaînages relâchés et les galons de bordure retirés. Ces derniers sont teints avec des colorants instables, ils seront donc lavés à part, puis recousus.

Il a été décidé de recourir à un lavage ${ }^{12}$ car les œuvres présentaient un encrassement très important, ainsi que des déformations qu'il était souhaitable d'atténuer avant de procéder à la conservation. Les risques de dégorgement ou de changement de dimension pouvaient quant à eux être maîtrisés grâce à un équipement perfectionné et à un suivi attentif du lavage. Des analyses colorimétriques réalisées avant et après le lavage devaient nous permettre d'évaluer l'efficacité de cette intervention. Lors du lavage, une brume composée d'eau déminéralisée et de tensioactif non ionique est aspirée à travers la tapisserie placée sur une immense table microperforée. La poussière est entrainée par le flux hors du textile, et ponctuellement un tamponnage à l'éponge facilite l'élimination des saletés. Des rinçages répétés à l'eau déminéralisée éliminent les restes du bain de lavage. Ces différentes étapes sont suivies par un contrôle régulier du $\mathrm{pH}$, de la conductivité de l'eau, de la température, et visuellement par des prélèvements des eaux évacuées pour suivre la progression du processus. À l'issue du lavage, la tapisserie est laissée à sécher sous un flux d'air tiède. L'ensemble du processus prend environ une journée. Après le lavage, les tapisseries redeviennent effectivement plus souples et offrent une surface plus plane. Les résultats des analyses colorimétriques présentés plus loin confirment quant à eux la sensation de coloris plus vifs et plus contrastés.

Les tapisseries ont alors été consolidées dans leurs zones de faiblesse par des pièces en lin teint placées au revers et fixées par des lignes de points verticales de façon à apporter localement le soutien requis. Ces pièces ont ensuite servi de support à un réseau de points de restauration ${ }^{13}$ qui ont maintenu et stabilisé le tissage original dans ces zones fragiles. Les tissus et les fils de restauration ont été teints avec des colorants naturels ${ }^{14}$ par Cécilia Aguirre. L'emploi de ces colorants a permis de rester proche en tons et en nature des colorants originaux et de s'approcher de la tenue des teintures de départ.

Nous avons ensuite procédé à un nouveau doublage de toutes les tapisseries. Cette étape est au cour du travail de conservation entrepris, car elle assure un soutien général et une protection contre la poussière et les frottements des supports de présentation. La doublure choisie est une toile de lin naturel décatie et lavée, légère, dense et stable face aux 
variations climatiques. Elle a été mise en place et cousue par des lignes ${ }^{15}$ verticales (sens de la tombée du tissu), disposées en quinconce de manière régulière. Aucun noud n'a été effectué pour éviter les points de contrainte. Le réseau est dense (fig. 2), on compte en moyenne 18 lignes par mètre carré.

Les tapisseries ont également été équipées d'un nouveau système de suspension avec un velcro débordant ${ }^{16}$ qui permet de répartir le poids de façon uniforme sans rigidifier ni contraindre le tissage en partie haute. Pour plus de discrétion, cette bande de velcro a été habillée d'un tissu reprenant la couleur des supports de présentation.

Ces supports sont des plans inclinés à $5^{\circ}$, habillés d'un velours bouclé de coton très fin, disposé de façon à retenir la tapisserie. L'objectif de cette installation est de limiter au maximum les tensions exercées sur les tapisseries lors de leur accrochage.

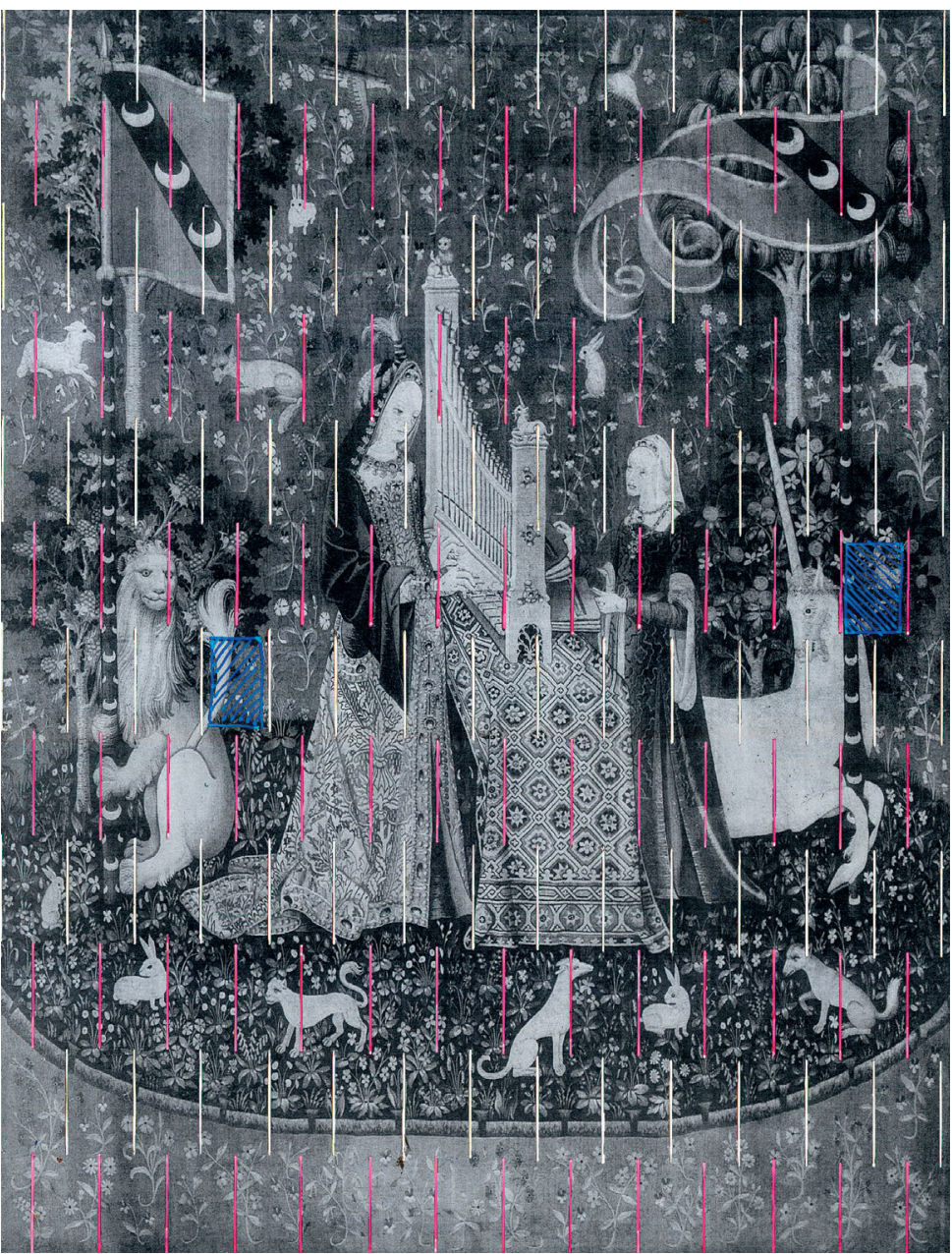

Fig. 2. Schéma de la répartition des lignes de fixation (en rose) de la doublure pour la tapisserie «L'Ouïe ». En bleu, les fenêtres d'observation aménagées pour accéder localement au revers. (c) Raphaëlle Déjean.

\section{Évaluation colorimétrique de l'état de la tapisserie et du nettoyage}

L'évaluation de la couleur par colorimétrie apporte des informations quantitatives sur les caractéristiques chromatiques de la surface colorée de l'œuvre textile. Elle dépend des propriétés des matériaux constitutifs - la nature du colorant utilisé, mais aussi celle de la fibre (présence ou non de pigmentation) et de son état de conservation (jaunissement) -, ainsi que des propriétés des matériaux apportés : nature et quantité de poussières et de taches.

La couleur des tapisseries de La Dame à la licorne a été évaluée à deux moments du programme de conservationrestauration : avant et après le nettoyage. Ces deux campagnes avaient pour objectif l'appréciation de l'effet du nettoyage (par comparaison des observations recueillies avant et après cette étape du traitement), ainsi que la constitution d'un point de référence (après le traitement) pour le suivi de l'évolution de leur état chromatique dans l'avenir. Ce deuxième point devrait permettre d'évaluer la vitesse de reprise de l'empoussièrement et de la diminution de l'intensité des couleurs des tapisseries dans le temps, afin de juger de la nécessité éventuelle de modifications concernant les conditions d'exposition ou d'un nouveau nettoyage. Si l'on considère que les couleurs visibles au revers n'ont pratiquement pas été dégradées par la lumière, on peut alors apprécier la dégradation des coloris de la face. Du fait de la présence des colorants synthétiques, en général moins stables à la lumière et au lavage que les colorants naturels d'origine, il convient également de surveiller une potentielle augmentation de l'écart chromatique, suite au lavage, entre les parties originales et les parties retissées.

\section{Approche méthodologique}

Pour mener à bien cette étude, nous avons eu recours à la colorimétrie - une méthode de mesure des couleurs standardisée, objective et quantitative, fondée sur trois valeurs : teinte, saturation et luminosité. Le résultat de chaque mesure comporte trois valeurs de coordonnées d'espace chromatique $L^{*} a^{*} b^{*}$, qui caractérisent la couleur mesurée. $L^{*}$ correspond à la luminosité ou balance noir (0)/blanc (100), $a^{*}$ correspond à la balance rouge $\left(+a^{*}\right) /$ vert $\left(-a^{*}\right)$ et $b^{*}$ à la balance jaune $\left(+b^{*}\right) /$ bleu $\left(-b^{*}\right)$. Les mesures ont été effectuées avec le spectrophotomètre CM-2500d (Konika Minolta Optics Inc.), muni d'une fenêtre de mesure d'un diamètre de $8 \mathrm{~mm}$. L'appareil était réglé en mode SCE (composante spéculaire exclue) et utilisé après une calibration avec des étalons noir et blanc à chaque mise en route de système. Pour le pilotage de l'appareil, acquisition et pré-traitement des données, nous avons utilisé le logiciel SpectraMagic XN (Konika Minolta Optics Inc.). Le traitement statistique des données a été fait avec le tableur Excel (Microsoft Corp.).

Pour caractériser chacune des six tapisseries, une gamme de couleurs et de nuances la plus large possible a été mesurée, incluant à chaque fois des parties originelles et des parties 


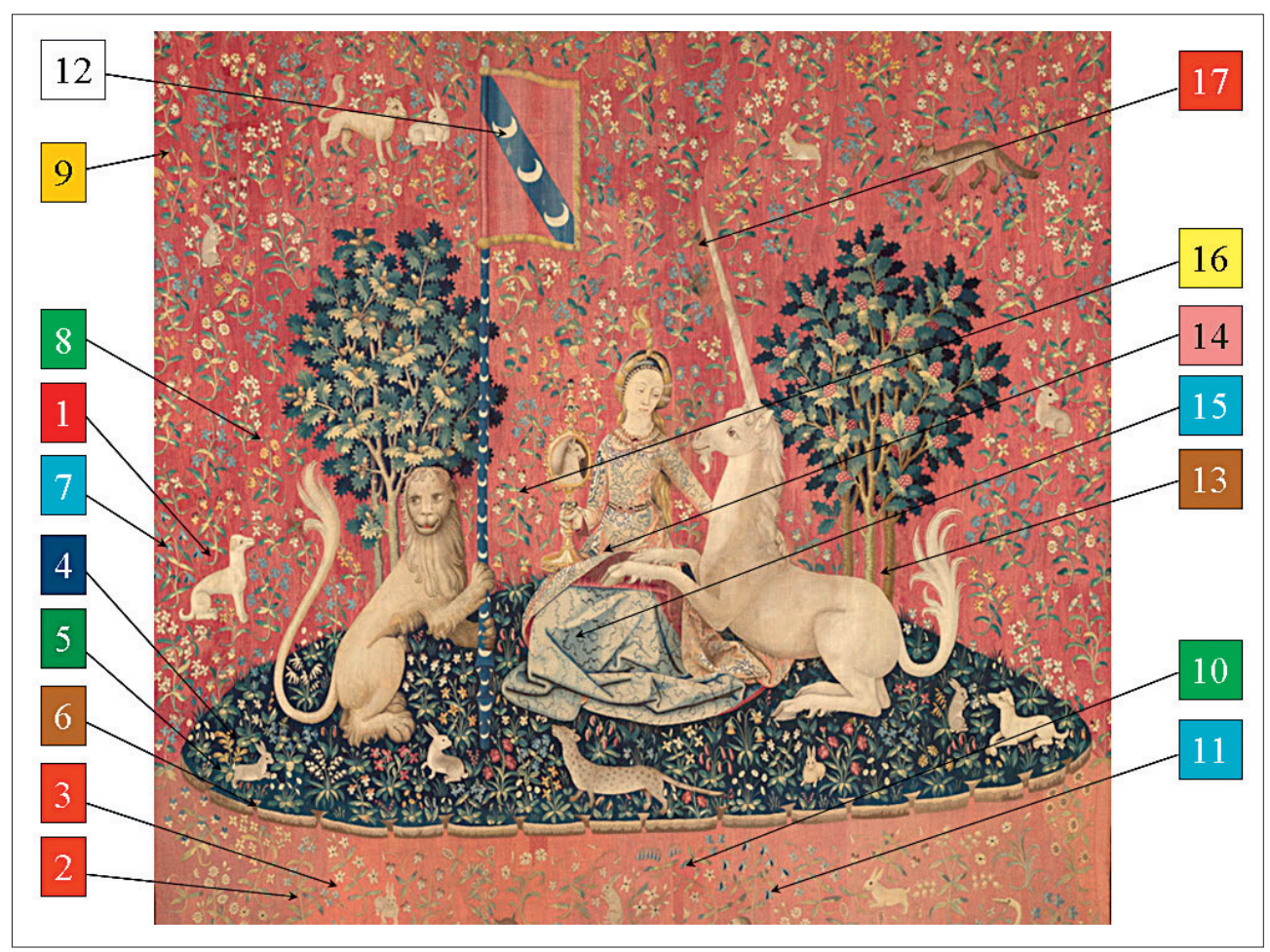

Fig. 3. Exemple de répartition des prises de mesures colorimétriques. Tapisserie

"La Vue ». Les couleurs de fond des numéros de mesures correspondent aux couleurs échantillonnées.

(C) Raphaëlle Déjean.

restaurées (fig. 3), tout en privilégiant les surfaces les plus homogènes. Chaque point de mesure est la valeur moyennée de trois prises successives faites automatiquement par l'appareil sans déplacement de sa tête de mesure. Pour diminuer l'erreur due au positionnement de la tête de mesure, plus ou moins reproductible, 2 à 4 points de mesure rapprochés par fragment de surface ont été sélectionnés. Ils permettaient de calculer les valeurs moyennes représentatives pour les surfaces choisies (fig. 4).

Les croissants blancs de gonfanons, qu'on retrouve sur toutes les tapisseries, sont des zones de tissage suffisamment

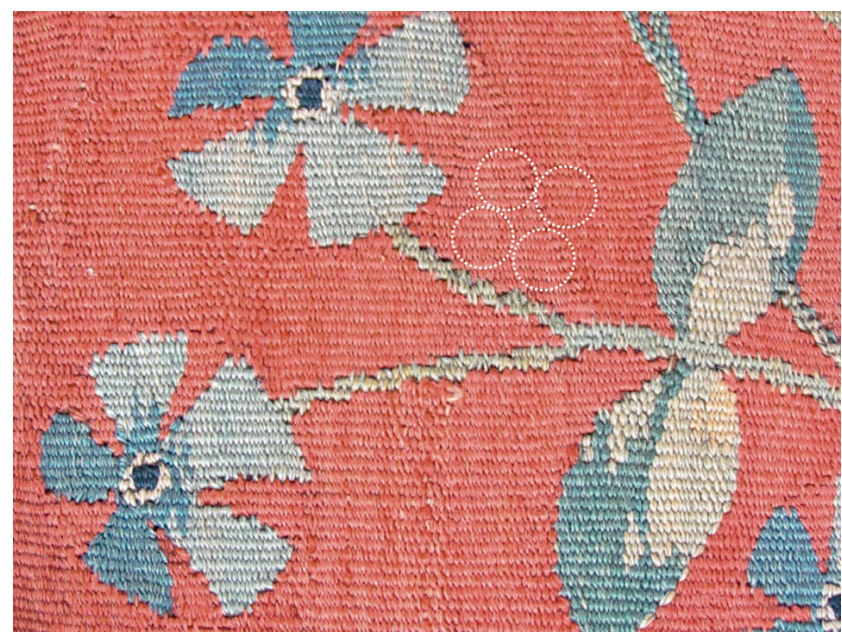

Fig. 4. Exemple de prises de mesures multiples. Fond de la tapisserie "L'Odorat ». Les cercles blancs correspondent aux surfaces de points des prises de mesures. ( ) Raphaëlle Déjean. grandes et homogènes. Ils ont été choisis pour servir de base de référence pour l'évaluation de l'empoussièrement de la tapisserie au cours du temps. L'achromaticité du blanc permet d'établir un classement par rapport à la luminosité. Pour être le plus représentatif possible, les points de mesure ont recouvert toute la surface du croissant (fig. 5).

Les mesures ont porté sur une vingtaine de nuances (recto et verso) sur chaque tapisserie, ce qui a représenté environ 120 points de mesure avant et après le nettoyage. La mise en œuvre de ce travail a nécessité l'immobilisation de chaque tapisserie pour une journée.

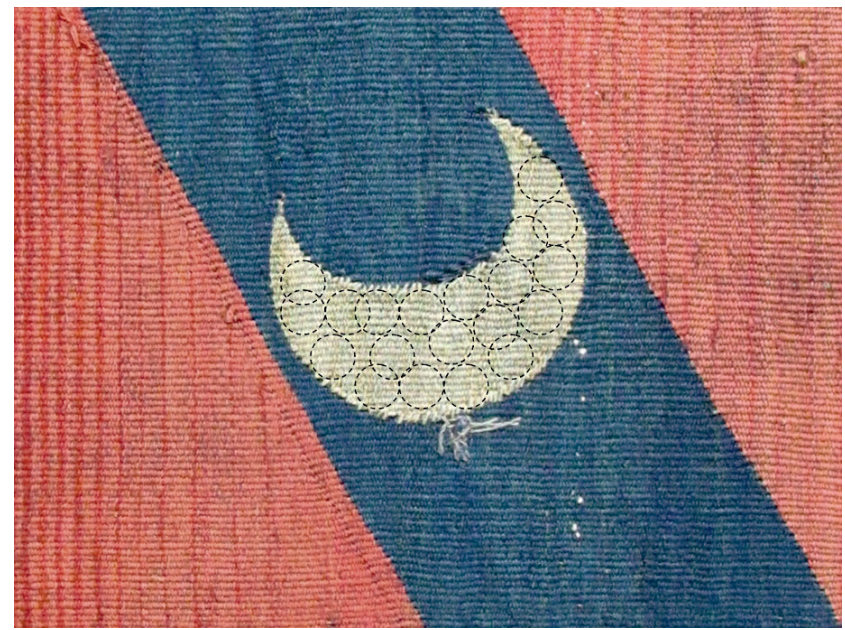

Fig. 5. Exemple de prises de mesures multiples sur les croissants : un gonfanon de la tapisserie "À mon seul désir ». Les cercles noirs correspondent aux surfaces de points des prises de mesures. () Raphaëlle Déjean. 


\section{Les résultats et leur interprétation}

La grande quantité de données accumulées durant cette étude permet des interprétations fines et spécifiques sur chaque tapisserie. Ici, nous présenterons uniquement la synthèse des résultats permettant d'évaluer l'impact du nettoyage.

Les diagrammes de luminosité $\left(L^{*}\right)$ ont été tracés à partir des valeurs moyennes de mesure des croissants blancs au recto (fig. 6a) et au verso (fig. 6b), avant et après nettoyage. La luminosité donne une indication sur le degré de clair ou d'obscur de la couleur. Plus la couleur est sombre, plus la luminosité est faible. On constate un gain de luminosité $L^{*}$ de 5,0 en moyenne sur la face. Dans tous les cas, avant ou après lavage, les valeurs de luminosité sont plus importantes sur la face que sur le revers. Cela peut s'expliquer par le palissement des couleurs, plus exposées à la lumière, et par une moindre accumulation de salissures due à l'entretien, probablement plus fréquent que celui du revers.

L'influence du nettoyage sur l'aspect chromatique des tapisseries a été évaluée à partir des diagrammes $d a^{*} / d b^{*}$ (fig. 7), qui correspondent pour chaque point à la différence des valeurs après et avant nettoyage.

Lorsque, sur l'axe rouge/vert, les valeurs sont positives $\left(+d a^{*}\right):$ l'échantillon est plus rouge ou moins vert, et lorsqu'elles sont négatives (- $\left.d a^{*}\right)$, l'échantillon est plus vert ou moins rouge. Lorsque, sur l'axe jaune/bleu, les valeurs sont positives $\left(+d b^{*}\right)$, l'échantillon est plus jaune ou moins bleu, et lorsque les valeurs sont négatives (- $\left.d b^{*}\right)$, l'échantillon est plus bleu ou moins jaune.

L'une des principales préoccupations étant une éventuelle augmentation du contraste chromatique entre les fils teints avec les colorants naturels et ceux teints avec les colorants synthétiques, nous avons reporté ces informations sur des diagrammes comparatifs.
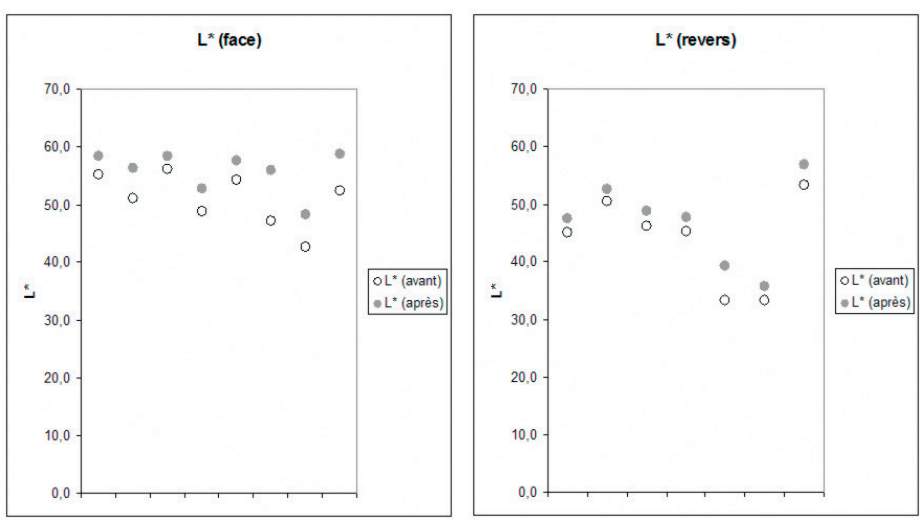

Fig. 6. Valeurs de luminosité $\left(L^{*}\right)$ obtenues sur les croissants de gonfanons (références du blanc) avant et après le nettoyage sur les faces (a) et les revers (b) de tapisseries.

Dans le groupe de jaunes et bruns (fig. 7a), la tendance générale est un gain de saturation pour les jaunes ( « $\mathrm{J}$ ») $\left(+d b^{*}\right)$, aussi bien pour les parties originales $(«(\mathrm{O}) »)$ que pour celles qui ont été restaurées («(R)»). Pour les bruns (« $\mathrm{B} »)$, les changements sont moins marqués. Ils oscillent autour de valeur $d b^{*}$ et $d a^{*}$ entre - 1 et 1 , ce qui rend difficile l'appréciation de la différence à l'œil nu. Néanmoins, deux bruns originaux semblent perdre un peu de leur composante rouge $\left(-d a^{*}\right)$, à la différence des autres points de mesure.

Dans le groupe de rouges et orange (fig. 7b), le gain de saturation semble globalement plus faible, sauf pour un rouge original dont le gain de saturation en rouge dépasse la valeur de 5,0 et en jaune, est proche de 3,0. Pour la majorité de points mesurés pour les rouges et orange, les gains chromatiques sont peu significatifs. Six sur dix se trouvent dans le disque délimité par coordonnées $d a^{*}$ et $d b^{*}$ entre - 1,0 et 1,0 .
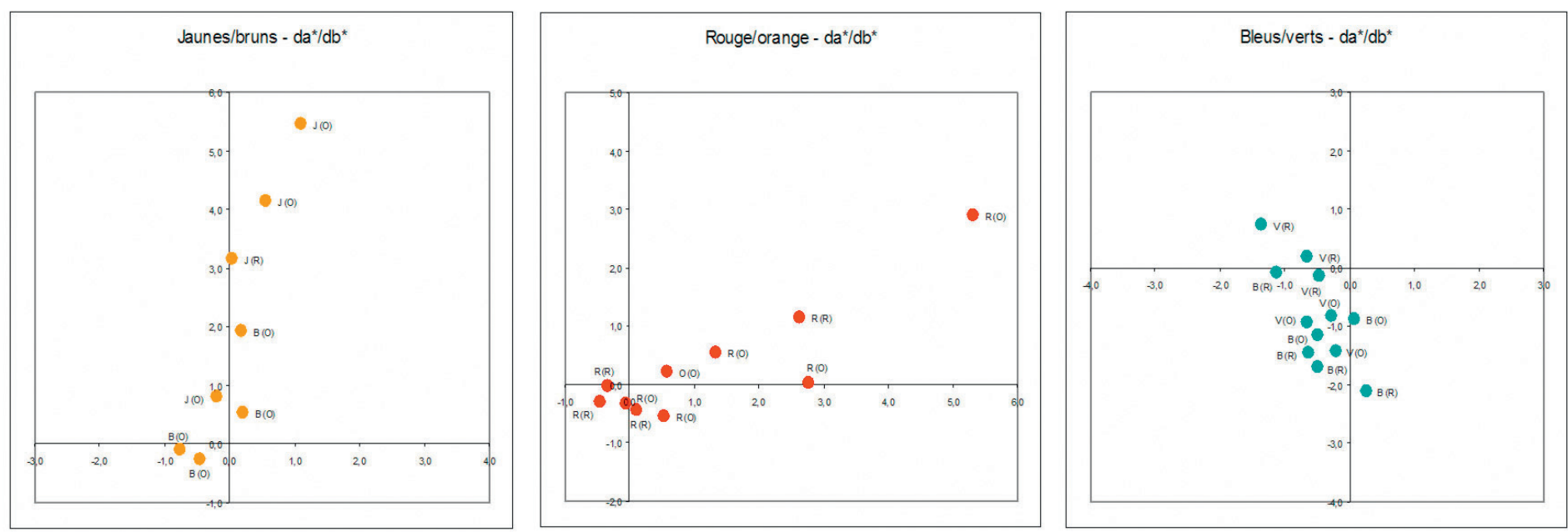

Fig. 7. Changement de teinte et de saturation $\left(d a^{*} / d b^{*}\right)$ des points mesurés sur la face des tapisseries par groupes de couleurs : (a) jaunes «J" et bruns « $\mathrm{B}$ », (b) rouges « $\mathrm{R}$ » et orange « $\mathrm{O}$ », (c) bleus « $\mathrm{B}$ » et verts « $\mathrm{V}$ ». Les prises de mesures colorimétriques dans les parties originelles sont marquées « $(\mathrm{O})$ » et dans les parties restaurées « $(\mathrm{R})$ ». L'axe horizontal représente les tendances vert (- $\left.d a^{*}\right) /$ rouge $\left(+d a^{*}\right) ; 1$ 'axe vertical représente les tendances bleu $\left(-d b^{*}\right) /$ jaune $\left(+d b^{*}\right)$. 
Les bleus et les verts (fig. 7c) présentent un comportement similaire après le nettoyage. Nous pouvons remarquer que le groupe des couleurs d'origine montre une perte de $d b^{*}$ autour de - 1,0. Les bleus et les verts bleuissent de manière systématique. Pour les couleurs des parties restaurées, deux tendances sont observées. Pour un groupe, le changement s'opère autour de - 1,0 $d a^{*}$ en tirant vers les verts et pour un autre, le changement est autour de - 2,0 d $b^{*}$, marquant un verdissement plus prononcé que pour les couleurs originales.

Mis à part quelques exemples de jaunes et un rouge, les gains chromatiques sont globalement plus faibles que les changements de luminosité observés sur les blancs, avec $d L^{*}$ autour de 5,0. Toutefois, ces changements allant globalement vers la saturation des couleurs, contribuent à l'augmentation des couleurs et du contraste chromatique.

De ces observations, il résulte que le comportement des couleurs des parties originelles et celui des couleurs des parties restaurées ne semblent pas très différents et que le contraste chromatique entre ces parties a été préservé.

\section{Conclusion}

Cette vaste opération de conservation-restauration permet de présenter au public des tapisseries qui ont retrouvé de l'éclat, dont l'aspect est plus propre, plus régulier et plus homogène, ce qui met en valeur leur iconographie et la qualité de leur tissage. Les plans inclinés sur lesquels elles reposent leur apportent de bonnes conditions pour une exposition durable.

D'un point de vue structurel, leur tissage est à la fois renforcé et soutenu. Le lavage a redonné souplesse et brillance aux fibres dont les coloris ressortent plus lumineux et plus saturés - les tapisseries paraissent actuellement plus vives.

Les mesures colorimétriques ont permis de décrire les tendances des changements observés $\left(d L^{*}\right.$ et $\left.d a^{*} / d b^{*}\right)$ de manière globale. L'on relève également une plus grande homogénéité d'aspect entre les tapisseries. Néanmoins, les différences de gains de luminosité et de teinte/saturation sont parfois assez marquées entre les couleurs du même groupe, car les mesures ont été prises dans des endroits présentant différents niveaux d'encrassement et différents degrés d'efficacité du nettoyage.

Les valeurs $L^{*}, a^{*}$ et $b^{*}$ ont été enregistrées pour tous les points de mesure qui ont été minutieusement localisés et documentés en constituant un référentiel. Ces informations permettront d'évaluer régulièrement l'état de surface de la tenture : son degré d'empoussièrement et de décoloration pourra être apprécié et donner lieu à des ajustements concernant les préconisations d'exposition.

La rénovation de la muséographie a également permis de concevoir des supports de présentation adaptés et de revoir les conditions d'éclairage et de contrôle du climat.

\section{Remerciements}

À toute l'équipe de restauration : Cécilia Aguirre (metzlicecilia@ gmail.com), Thalia Bajon-Bouzid (thbajonbouzid@gmail.com), Anne Breugnot (annebreugnot@yahoo.fr), Sylvie Forestier (sylforestier@orange.fr).

Nous voudrions également remercier Athénaïs Davantès (stagiaire Master 2) pour son aide dans la réalisation de plusieurs mesures colorimétriques.

\section{Notes}

1. Depuis sa formulation par Kendrick en 1921, tandis que l'interprétation globale a bénéficié de nombreux travaux

(Nordenfalk, 1976, Erlande-Brandenburg, 1978, Boudet, 2000).

2. Kendrick, 1921, p. 662-666 ; Reynaud, 1993, p. 265-270 ; Nordenfalk, 1976 , p. 25-28 ; Nordenfalk, 1982, p. 53-56; Erlande-Brandenburg, 1978 ; Boudet, 2000, p. 551-553. Synthèses et bibliographie dans Joubert, 1987 ; Joubert et Huchard, 2002 ; Delahaye, 2012.

3. Sa nomination à la charge de premier président du parlement de Paris en 1523 marque le sommet de l'ascension sociale des Le Viste. Voir notamment Vaivre, 1984, p. 397-434 ; Souchal, 1983, p. 209-267. Pour le rapprochement entre la tapisserie et le vitrail de Saint-Germain l'Auxerrois, voir Decu Teodorescu, 2010, p. 355-367. Pour l'attribution de la commande à Antoine II peu avant ou au moment de son mariage avec Jacqueline Raguier, voir déjà Nordenfalk, 1982
4. Pour les différentes interventions, voir Joubert, 1987.

5. L'entrecroisement des fils au revers prouve que le tissage a été réalisé de gauche à droite. Lors de l'exposition, la tapisserie est présentée avec les fils de chaîne placés à l'horizontale, mais sur le métier les fils de chaîne étaient verticaux et le tissage a commencé par le bord gauche. En moyenne, on a une réduction de 5,5 fils de chaîne au $\mathrm{cm}$ et de 20 à 44 coups de trame au $\mathrm{cm}$ selon les zones.

6. Emploi de relais décoratifs, de tissages en forme, de battage de couleurs, et grand soin apporté aux finitions au revers.

7. Environ trente coloris.

8. Dans ces tapisseries, tous les colorants ont été identifiés par chromatographie en phase liquide, avant le nettoyage par W. Nowik (LRMH). Rapport en cours de rédaction.

9. Mis en évidence également par chromatographie en phase liquide.

10. En fonction de leur emplacement et du support.

11. Réseau de coutures croisées en diagonale.
12. Décision prise le 17 avril 2012 par comité scientifique : Marie Lavandier (C2RMF) - présidente, Élisabeth TaburetDelahaye (musée de Cluny), Roberta Cortopassi (C2RMF), Raphaëlle Déjean (restauratrice textile), Sylvie Forestier (restauratrice textile), Dominique de Reyer (LRMH), Regula Shorta (Fondation Abegg), André Brutillot (Musée national de Munich), Thierry Crépin-Leblond (château d'Écouen), Amaury Lefebure (domaine de Malmaison), Witold Nowik (LRMH), Pierre Provoyeur (SMF). Le lavage a été effectué à la Manufacture royale De Wit (Malines, Belgique).

13. Points dérivés d'un point de broderie appelé «point de Boulogne ».

14. Emploi d'extraits secs de garance, gaude, indigo, myrobolan et châtaignier.

15. Lignes longues de $30 \mathrm{~cm}$ et espacées de $20 \mathrm{~cm}$ sur une rangée.

16. Le velcro est cousu sur une bande de lin et placé au-dessus de la tapisserie. La bande de lin est cousue à la doublure au revers sur environ $20 \mathrm{~cm}$ de hauteur pour une meilleure répartition du poids. 


\section{Références bibliographiques}

Boudet J.-P., 2000, «Jean Gerson et La Dame à la licorne ", dans Religion et Société urbaine au Moyen Âge, études offertes à Jean-Louis Biget, Paris, p. 551-553.

Decu Teodorescu C., 2010, « La tenture de La Dame à la licorne. Nouvelle lecture des armoiries ", Bulletin monumental, t. 164 , p. 355-367.

Delahaye E., 2012, La Dame à la licorne, Paris. Erlande-Brandenburg A., 1978, La Dame à la licorne, Paris.

Joubert F., 1987, La Tapisserie médiévale au musée de Cluny, Paris.

Joubert F., Huchard V., 2002, La Tapisserie médiévale au musée de Cluny, Paris.
Kendrick A. F., 1921, «Quelques remarques sur les tapisseries de La Dame à la licorne du musée de Cluny », Actes du Congrès d'histoire de l'art, Paris, t. III, p. 662-666.

Nordenfalk C., 1976, "Les cinq sens dans l'art du Moyen Âge ", Revue de l'Art, $n^{\circ} 34$, p. 25-28

Nordenfalk C., 1982, «Qui a commandé les tapisseries dites de La Dame à la licorne? ", Revue de l'Art, n 55, p. 53-56.

Reynaud N., 1993, «Le Maître des Très Petites Heures d'Anne de Bretagne ", dans cat. exp. Les manuscrits à peintures en France 1440-1520, dir. François Avril et Nicole Reynaud, Paris, Bibliothèque nationale de France, p. 265-270.
Souchal G., 1983, « Messeigneurs Les Vistes et La Dame à la licorne », Bibliothèque de l'École des Chartes, t. 141, p. 209267.

Vaivre J.-B. (de), 1984, « Messire Jehan Le Viste, chevalier, seigneur d'Arcy et sa tenture au lion et à la licorne ", Bulletin monumental, t. 142, p. 397-434. 